\title{
Fatores Associados à Manutenção do Vício de Fumar e do Consumo de Álcool entre Acadêmicos de Medicina em uma Capital do Nordeste do Brasil
}

Factors Associated with Continued Smoking Addiction and Alcohol Consumption among Medical Students in Capital City in the Northeast of Brazil

\section{PALAVRAS-CHAVE}

- Hábito de Fumar.

- Alcoolismo.

- Estudantes.

- Medicina.

Ileana Pitombeira Gomes ${ }^{I}$ Renata Allana da Costa Pereira ${ }^{I}$

Brena Ferreira dos Santos ${ }^{I}$

Marcelo de Almeida Pinheiro II

Carlos Henrique Alencar ${ }^{\mathrm{I}}$

Luciano Pamplona de Góes Cavalcanti ${ }^{I}$

\section{RESUMO}

Introdução: Há um aumento no consumo de drogas entre os jovens no Brasil. Esse consumo se destaca entre os estudantes universitários, acarretando uma preocupação adicional quando associado aos estudantes da área da saúde. Objetivo: Identificar os fatores associados à manutenção do vício de fumar e do consumo de álcool entre acadêmicos de Medicina. Métodos: Trata-se de um estudo analitico, de prevalência, envolvendo estudantes de Medicina das quatro escolas médicas da cidade de Fortaleza, Nordeste do Brasil. Foi aplicado um questionário semiestruturado, contendo 46 perguntas objetivas, aos estudantes que cursavam o primeiro ano (S1/S2) durante o ano de 2012 e novamente em 2016, quando esses mesmos estudantes se encontravam no internato (I3/I4). A amostra foi calculada considerando como população do estudo o número máximo de alunos nos dois períodos avaliados.O projeto foi aprovado pelo CEP, por meio do Parecer $n^{o}$ 020/2012. Resultados: Foram entrevistados 360 estudantes no primeiro momento da pesquisa e 354 estudantes no segundo momento. O consumo de tabaco passou de $17,4 \%$ durante o primeiro ano do curso para $28,2 \%$ durante o internato $(p<0,001)$. O mesmo ocorreu com o consumo de álcool, que já era elevado no início do curso $(84,6 \%) e$ aumentou para $92,6 \%$ ( $p<0,001)$. No primeiro ano do curso, 40,5\% dos estudantes referiram já ter se embriagado pelo menos uma vez. Durante a faculdade, esse percentual subiu para $59,5 \%$ ( $R P=1,66$; $p<0,001)$. Conclusão: O consumo de álcool e tabaco aumentou de forma importante durante o curso de Medicina. Há necessidade de intervenções nos hábitos dos acadêmicos de Medicina com o objetivo de reduzir o consumo exagerado de álcool e a manutenção do tabagismo nesta população. 


\section{KEY-WORDS}

- Smoking.

- Alcoholism.

- Students.

- Medicine.

\begin{abstract}
Introduction: Drug use among young people in Brazil is increasing, particularly among the university students, leading to additional health-related concerns for students. Objective: To identify factors associated with continued smoking addiction and alcohol consumption among medical students. Methods: An analytical, prevalence study was carried out involving medical students at the four medical schools of Fortaleza, northeastern Brazil. A semi-structured questionnaire with 46 objective questions was applied to students in their first year of the course (S1/S2) in 2012 and again in 2016, when these same students were taking their internship (I3/I4). The sample was calculated based on a study population consisting of all the students in the two periods evaluated. The project was approved by the REC, through opinion no. 020/2012.Results: 360 students were interviewed in the first phase of the research, and 354 students in the second phase. Tobacco consumption increased from $17.4 \%$ during the first year of the course to $28.2 \%$ during the internship ( $p<0.001)$. The same occurred with alcohol consumption, which was already high at the beginning of the course $(84.6 \%)$ and increased to $92.6 \%$ ( $p<0.001)$. In the first year of the course, $40.5 \%$ of the students reported having got drunk at least once. During their time at medical school, this percentage rose to 59.5\% $(P R=1.66, p<0.001)$. Conclusion: Alcohol and tobacco consumption increased significantly during medical school. There is a need for interventions in the habits of medical student, in order to reduce excessive alcohol consumption and smoking in this population.
\end{abstract}

Recebido em: 28/8/2018

Aceito em: 3/9/2018

\section{INTRODUÇÃO}

$\mathrm{O}$ abuso e a dependência de substâncias químicas, tais como álcool e tabaco, causam riscos políticos, sociais e econômicos. O consumo de drogas acomete a sociedade em todo o mundo, estabelecendo uma ameaça à saúde individual, ao bem-estar coletivo e ao desenvolvimento social ${ }^{1}$. O consumo de álcool e derivados do tabaco amplia os gastos com tratamentos e internações hospitalares, e aumenta indicadores como acidentes de trânsito, violência urbana e anos potenciais de vida perdidos devido ao aumento de mortes prematuras ${ }^{2}$.

$\mathrm{O}$ ingresso no meio universitário se caracteriza por uma nova etapa na vida de jovens estudantes. A chegada ao curso superior é um evento que permite novas experiências que surgem em encontros sociais e é uma fase de transição para a vida adulta, o que pode favorecer o acesso a drogas lícitas ou ilícitas ${ }^{1}$. Em virtude das mudanças em relação à autonomia de aprendizagem, o período inicial do curso superior é considerado de maior vulnerabilidade, sendo frequentemente associado ao consumo excessivo de substâncias químicas ${ }^{3}$.

Conhecer a prevalência e os fatores associados ao consumo de substâncias químicas entre estudantes tem sido um desafio perseguido nos últimos anos, principalmente entre os estudantes da área da saúde ${ }^{4}$. No caso dos estudantes do curso de Medicina, essa preocupação parece maior, considerando o risco de esse comportamento permanecer durante a formação e interferir no ambiente de trabalho, já que esses alunos serão promotores diretos da saúde para a população em geral ${ }^{5}$. Os estudantes de Medicina vêm exercendo um papel importante nos principais programas de prevenção e orientação do consumo de bebidas alcoólicas e tabaco, levando orientação à comunidade e exercendo seu papel social ${ }^{6}$. Desta forma, o objetivo deste trabalho foi identificar os fatores associados à manutenção do vício de fumar e do consumo de álcool entre acadêmicos de Medicina residentes na cidade de Fortaleza, Ceará, Brasil.

\section{MÉTODOS}

\section{Desenho do estudo}

Foi realizado um estudo de prevalência, analítico, envolvendo estudantes de Medicinadas quatro instituições de ensino superior que formam médicos da cidade de Fortaleza.

Foram incluídos estudantes regularmente matriculados nas quatro instituições quando cursavam o primeiro ano do curso (S1/S2) em 2012 e novamente quando se encontravam no internato (I3/I4), durante o ano de 2016.

\section{Amostra}

Foi considerada como população do estudo o número máximo de alunos nos dois períodos avaliados. Utilizou-se como parâmetro uma frequência esperada de $10 \%$ de pessoas fumantes, com erro de $3 \%$. Aos valores calculados foram acrescentados 
10\% como correção para possíveis perdas durante a coleta dos dados.

\section{Coleta de dados}

Foi aplicado um questionário estruturado, contendo 46 perguntas. Os itens se referiama características sociodemográficas, informações sobre consumo de tabaco e ingestão de álcool, e sobre como esses temas foram abordados na grade curricular das respectivas instituições.

Na primeira etapa (2012), o questionário foi aplicado em sala de aula. Já na segunda etapa, ocorrida em 2016, o mesmo questionário foi aplicado nos hospitais em que os alunos atuavam como internos ou em momentos presenciais nas respectivas instituições de ensino.

\section{Análise dos dados}

Os dados foram digitados utilizando-se o software Epi-info Versão 3.5.1, e a análise realizada com o software Stata 11.2. Foram utilizados testes paramétricos (teste $t$ de Student) e não paramétricos (Qui-Quadrado de Pearson e teste de Kruskal-Wallis) segundo as características das variáveis do estudo. Foram calculadas também as razões de prevalência e seus intervalos de confiança, e considerou-se uma significância de 95\%.

\section{Aspectos éticos}

Este estudo seguiu os princípios éticos de pesquisa envolvendo seres humanos da Resolução 466/12 do Conselho Nacional de Saúde e foi aprovado pelo Comitê de Ética em Pesquisa (CEP) da Unichristus (Protocolo nº 020/2012).

\section{RESULTADOS}

\section{Características da amostra no início do curso}

Entre os 472 estudantes matriculados nas quatros instituições e que cursavam o primeiro ano de Medicina, foram entrevistados 360 (76,3\%). Predominou o sexo feminino (198; 54,8\%), estudantes solteiros (352; 97,5\%), nascidos na cidade de Fortaleza $(240 ; 66,8 \%)$, residindo com os pais $(298 ; 82,7 \%)$ e com renda familiar superior a dez salários mínimos (102; 28,2\%). A maioria vinha de instituições privadas de ensino médio (208; 57,6\%), e, entre esses alunos, $128(61,5 \%)$ tinham bolsa (Prouni) ou financiamento (Fies) estudantis custeados pelo Ministério da Educação à época.

\section{Aspectos ligados ao consumo de tabaco e álcool}

Do total de alunos avaliados, 63 (17,4\%) já haviam fumado alguma vez na vida. E, destes, $71,4 \%$ residiam com pais. Curiosidade e influência de outros familiares fumantes foram as principais justificativas dadas pelos estudantes para o iní- cio do hábito de fumar. A maior parte dos estudantes $(84,2 \%)$ acreditava que acadêmicos de Medicina deveriam receber treinamento sobre técnicas de cessação do hábito de fumar durante a graduação. $\mathrm{O}$ maior consumo foi associado ao sexo masculino ( $R P=1,80 ; p=0,00)$. Por outro lado, esse consumo não esteve associado ao recebimento de algum tipo de financiamento $(p=0,810)$, presença de bolsa acadêmica $(p=0,08)$ ou mesmo o tipo de instituição de ensino $(\mathrm{p}=0,436)$.

Entre os estudantes que fumavam, a experimentação do álcool foi mais precoce. Eles se embriagaram pela primeira vez mais jovens, e o número de doses consumidas foi maior que para os estudantes que não fumavam $(\mathrm{p}<0,001)$ (Tabela 1$)$.

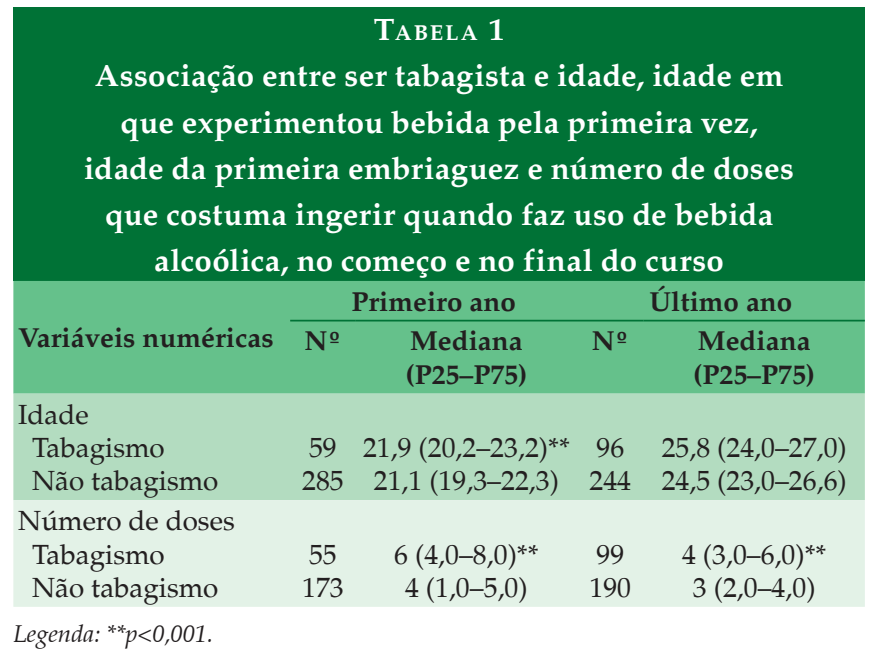

Informações sobre consumo excessivo e malefícios ocasionados pelo álcool foram recebidas por 95,1\% dos estudantes durante as aulas. Entretanto, tais informações foram insuficientes para interferir na mudança do hábito de beber excessivamente. Destaca-se que, ao serem questionados se deveriam receber treinamento específico sobre como desestimular o consumo excessivo de álcool, 83\% afirmaram a importância desse treinamento $(\mathrm{p}=0,014)$ (Tabela 2).

\section{Características socioeconômicas ao final do curso}

O consumo de bebida alcoólica aumentou 2,27 vezes, passando de $36,1 \%$ dos estudantes no primeiro ano do curso para $81,9 \%$ durante o internato. Permaneceu o consumo ocasional de bebida alcoólica, sendo as opções de bebidas alcoólicas mais consumidas a cerveja ou chope e a vodca. No início do curso, a maior parte dos alunos declarou continuar bebendo a mesma quantidade que bebia antes de se tornar universitária $(34,9 \%)$. Entretanto, ao serem questionados novamente no internato, a maior parte deles assumiu ter aumentado o consumo de álcool durante o curso superior (46,3\%) (Tabela 3). 


\begin{tabular}{|c|c|c|c|c|}
\hline \multirow{3}{*}{ Variáveis } & \multicolumn{4}{|c|}{$\begin{array}{l}\text { TABELA } 2 \\
\text { ol alguma vez na vida por estudantes do } \\
\text { urso de Medicina em Fortaleza (CE) }\end{array}$} \\
\hline & \multicolumn{2}{|c|}{ Primeiro ano } & \multicolumn{2}{|c|}{ Último ano } \\
\hline & $\mathrm{n} / \mathrm{N}(\%)$ & RP (IC) & $\mathrm{n} / \mathrm{N}(\%)$ & RP (IC) \\
\hline $\begin{array}{l}\text { Sexo } \\
\text { Masculino } \\
\text { Feminino }\end{array}$ & $\begin{array}{l}142 / 163(87,1) \\
161 / 197(81,7)\end{array}$ & $\begin{array}{c}1,06(0,97-1,16) \\
1\end{array}$ & $\begin{array}{l}140 / 155(90,3) \\
188 / 199(94,4)\end{array}$ & $\begin{array}{c}0,95(0,89-1,01) \\
1\end{array}$ \\
\hline $\begin{array}{l}\text { Financiamento } \\
\text { Sim } \\
\text { Não }\end{array}$ & $\begin{array}{l}108 / 127(85,0) \\
194 / 231(83,9)\end{array}$ & $\begin{array}{c}1,01(0,92-1,11) \\
1\end{array}$ & $\begin{array}{l}130 / 143(90,9) \\
198 / 211(93,8)\end{array}$ & $\begin{array}{c}0,96(0,91-1,03) \\
1\end{array}$ \\
\hline $\begin{array}{l}\text { Bolsista } \\
\text { Sim } \\
\text { Não }\end{array}$ & $\begin{array}{c}7 / 7(100,0) \\
292 / 34(83,6)\end{array}$ & $\begin{array}{c}1,19(1,14-1,25) \\
1\end{array}$ & $\begin{array}{c}82 / 88(93,1) \\
244 / 264(92,4)\end{array}$ & $\begin{array}{c}1,01(0,94-1,07) \\
1\end{array}$ \\
\hline $\begin{array}{l}\text { Instituição de ensino } \\
\text { Particular } \\
\text { Pública }\end{array}$ & $\begin{array}{l}176 / 207(85,0) \\
127 / 153(83,0)\end{array}$ & $\begin{array}{c}1,02(0,93-1,12) \\
1\end{array}$ & $\begin{array}{l}177 / 193(91,7) \\
119 / 124(95,9)\end{array}$ & $\begin{array}{c}0,95(0,90-1,01) \\
1\end{array}$ \\
\hline $\begin{array}{l}\text { Recebeu informações sobre os males do excesso de álcool } \\
\text { Sim } \\
\text { Não }\end{array}$ & $\begin{array}{c}222 / 257(86,3) \\
71 / 86(82,5)\end{array}$ & $\begin{array}{c}1,04(0,93-1,16) \\
1\end{array}$ & $\begin{array}{c}291 / 306(95,1) \\
32 / 35(91,4)\end{array}$ & $\begin{array}{c}1,04(0,93-1,15) \\
1\end{array}$ \\
\hline $\begin{array}{l}\text { Estudantes devem receber treinamento para reduzir o consumo } \\
\text { de álcool } \\
\text { Sim } \\
\text { Não }\end{array}$ & $\begin{array}{c}270 / 325(83,0) \\
30 / 30(100,0)\end{array}$ & $\begin{array}{c}0,83(0,79-0,87)^{*} \\
1\end{array}$ & $\begin{array}{c}310 / 333(93,0) \\
17 / 17(100,0)\end{array}$ & $\begin{array}{c}0,93(0,90-0,95) \\
1\end{array}$ \\
\hline $\begin{array}{l}\text { Recebeu treinamento sobre consumo excessivo de álcool nas aulas } \\
\text { Sim } \\
\text { Não }\end{array}$ & $\begin{array}{c}37 / 41(90,2) \\
258 / 306(84,3)\end{array}$ & $\begin{array}{c}1,07(0,95-1,19) \\
1\end{array}$ & $\begin{array}{l}148 / 156(94,8) \\
180 / 195(92,3)\end{array}$ & $\begin{array}{c}1,02(0,97-1,085) \\
1\end{array}$ \\
\hline
\end{tabular}

Informações relacionadas ao consumo de bebidas alcoólicas por estudantes do primeiro e último ano do curso de Medicina em Fortaleza (CE)

Variáveis

Consumo atual de bebida alcoólica

$\begin{array}{ll}\text { Sim } & 124 \text { Não }\end{array}$

Frequência com que consome bebidas

Ocasionalmente

Pelo menos uma vez por mês

1 ou 2 vezes por semana

3 ou 4 vezes por semana

5 vezes por semana ou mais

Já se embriagou

Sim

Primeiro ano

Não

№ $\%$

$\%$

$\begin{array}{cccc}124 & & 277 & 81,95 \\ 220 & 36,0563,95 & 61 & 18,05\end{array}$

Principal tipo de bebida

Whisky

Vodca

Vinho

Cerveja/chope

Aguardente/cachaça

Rum

Outra

$\begin{array}{cccc}154 & 63,90 & 122 & 40,94 \\ 45 & 18,67 & 97 & 32,55 \\ 40 & 16,60 & 71 & 23,83 \\ 1 & 0,41 & 7 & 2,35 \\ 1 & 0,41 & 1 & 0,34 \\ & & & \\ 171 & 50,89 & 251 & 73,82 \\ 165 & 49,11 & 89 & 26,18\end{array}$

Com que frequência exagerou o consumo

Ocasionalmente

Mensalmente

Mais de uma vez no mês

Semanalmente

$\begin{array}{cccc}20 & 8,51 & 5 & 1,71 \\ 81 & 34,47 & 74 & 25,26 \\ 17 & 7,23 & 24 & 8,19 \\ 105 & 44,68 & 183 & 62,46 \\ 12 & 0,85 & 3 & 1,02 \\ - & - & - & - \\ 10 & 4,26 & 4 & 1,37 \\ & & & \\ 136 & 45,33 & 79 & 25,08 \\ 145 & 48,33 & 214 & 67,94 \\ 10 & 3,33 & 15 & 4,76 \\ 3 & 1,00 & 4 & 1,27 \\ 6 & 2,00 & 3 & 0,95 \\ & & & \\ 56 & 23,24 & 31 & 10,54 \\ 55 & 22,82 & 12 & 4,08 \\ 117 & 48,55 & 90 & 30,61 \\ 12 & 4,98 & 110 & 37,41 \\ 1 & 0,41 & 51 & 17,35 \\ & & & \\ 86 & 27,56 & 40 & 12,35 \\ 109 & 34,94 & 85 & 26,23 \\ 74 & 23,72 & 150 & 46,30 \\ 43 & 13,78 & 40 & 15,12\end{array}$

Qual o período em que era maior o consumo de bebida

Antes de se preparar para o vestibular

Ao estudar para o vestibular

Logo que entrou na faculdade

Durante o período intermediário da faculdade

Durante o período do internato

Houve mudança de hábito de beber após entrar na faculdade

Não, não bebia e continuo sem beber

Não, continuo bebendo a mesma quantidade

Sim, aumentei o consumo

Sim, diminuí o consumo

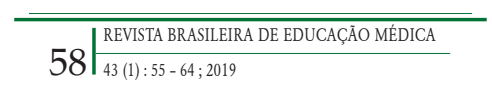


O consumo de tabaco esteve fortemente associado ao sexo masculino ( $R P=1,56 ; p=0,008)$ (Tabela 4). Esse consumo não foi associado ao fato de o aluno ter algum tipo de financiamento universitário, ser bolsista ou à origem da instituição de ensino $(p>0,05)$.

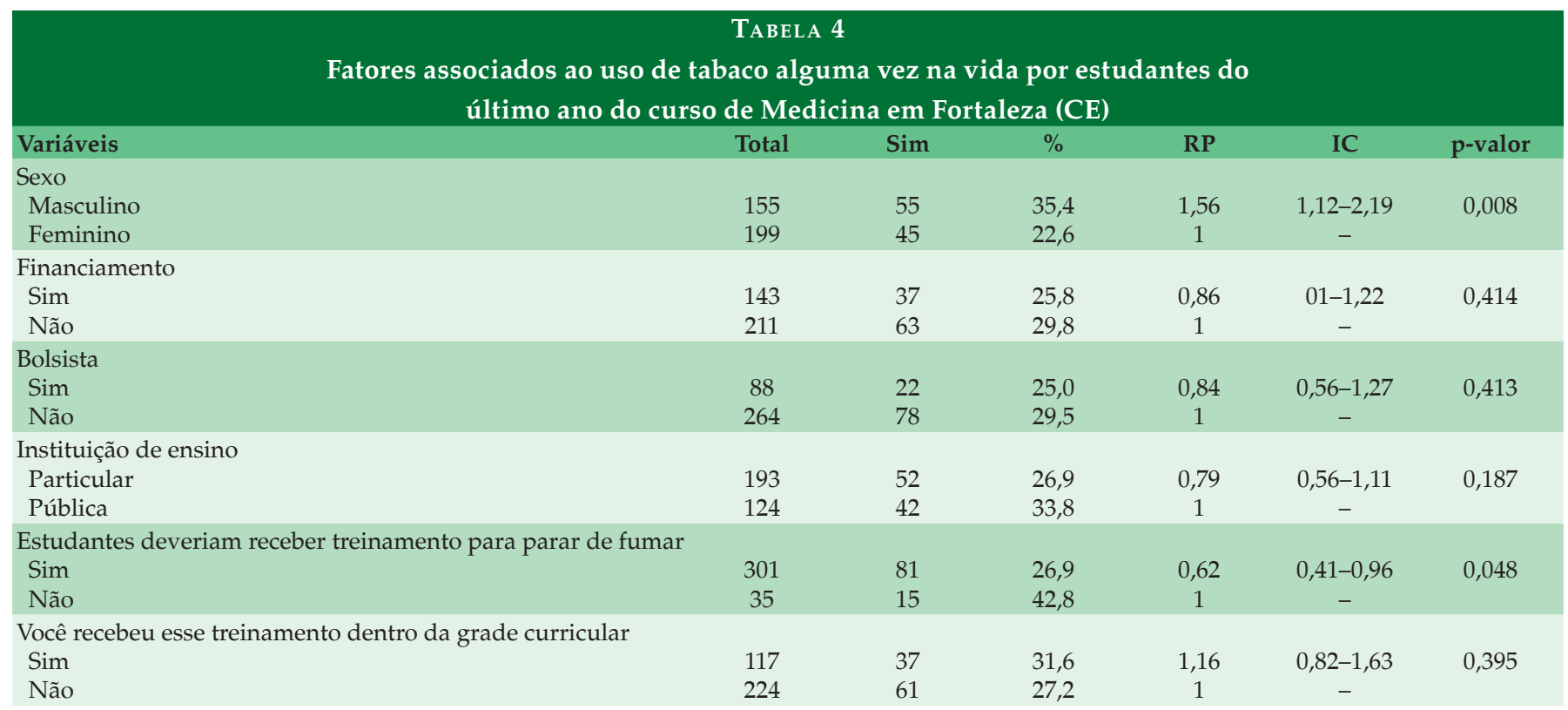

Entre os entrevistados, 73,1\% afirmaram que acadêmicos de Medicina deveriam receber treinamento sobre técnicas de cessação do hábito de fumar $(\mathrm{p}=0,048)$.

O hábito de fumar aumentou de $17,4 \%$ para $28,2 \%$ no final do curso, com $R P=1,62$ e p=0,001. Apenas um estudante afirmou fazer uso de tabaco no ambiente hospitalar ou arredores (universidade). Dos estudantes que fumavam no início do cur- so, $84,4 \%$ reconheceram a importância de receber treinamento especifico sobre técnicas de cessação do tabagismo durante as aulas. Esse resultado não diferiu de forma significativa quando comparado ao dos não fumantes, muito menos contribuiu para reduzir o hábito de fumar ou aumentar a prevalência de estudantes que tentaram parar de fumar (Tabela 5).

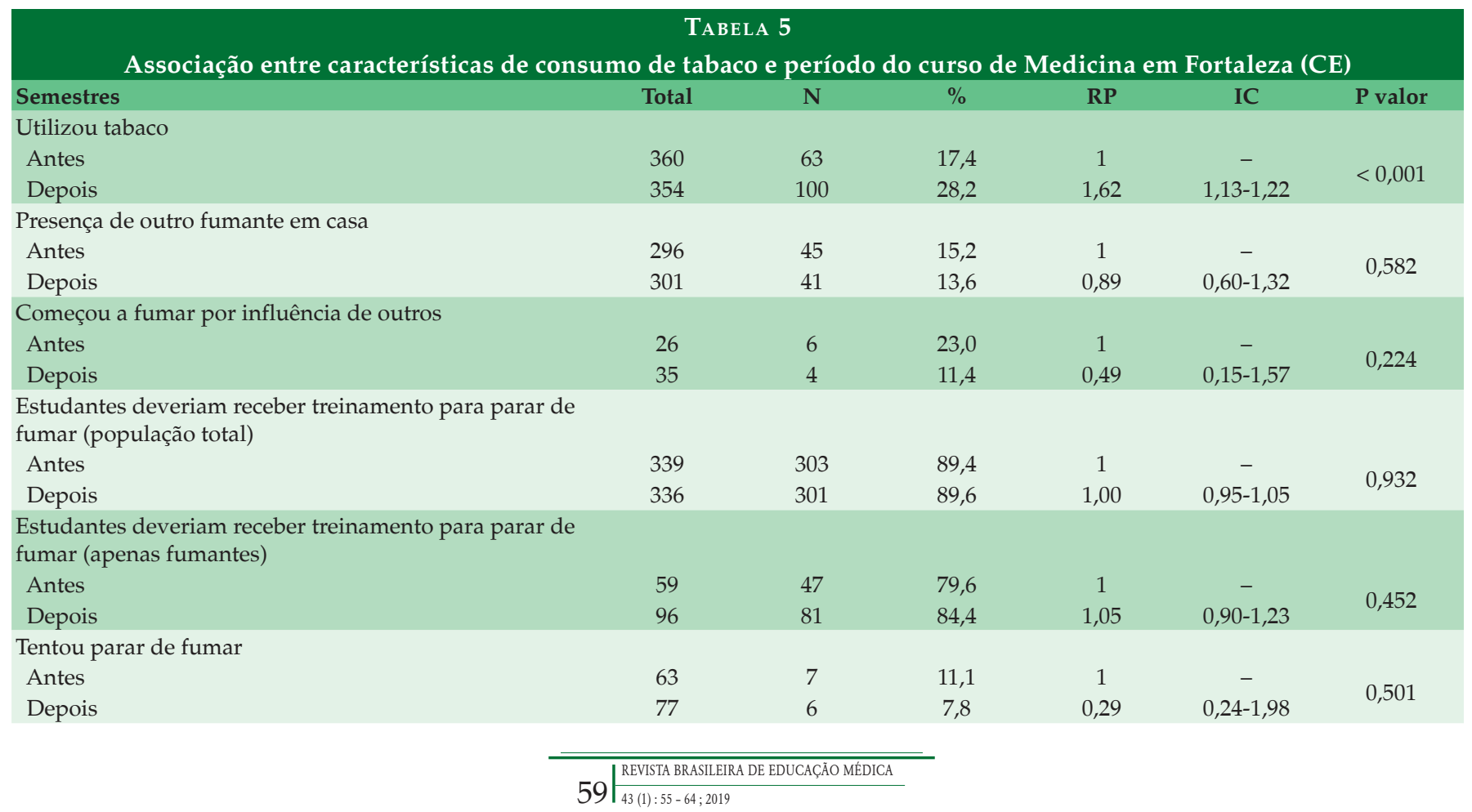


O consumo de bebida alcoólica foi de $82 \%$, mesmo ocasionalmente $(\mathrm{RP}=1,28 ; \mathrm{p}=0,000)$ (Tabela 6). Ao associarem episódios de embriaguez, 59,5\% relataram tal excesso $(p=0,000)$. A embriaguez esteve presente em todos os momentos de lazer questionados aos estudantes, sendo mais frequente em festas de faculdade e finais de semana, apresentando valores estatisticamente significantes.
A percepção em relação ao consumo de bebida alcoólica aumentou para mais de $42 \%$, apresentando significância estatística $(p=0,000)$ e prevalência de 2,06. Destaca-se o aumento significativo no consumo de álcool entre os estudantes. $\mathrm{O}$ alto valor do início do curso $(84,6 \%)$ cresceu mais ainda, alcançando $92,6 \%(p<0,000)$. Houve aumento expressivo também no número de alunos que permaneceram consumindo

\begin{tabular}{|c|c|c|c|c|c|c|}
\hline & $\begin{array}{l}\text { TABEL } \\
\text { álcool }\end{array}$ & fodo & so de & ina er & rtaleza (C & \\
\hline Semestre & Total & $\mathbf{N}$ & $\%$ & RP & IC & p-valor \\
\hline Consumiu & & & & & & \\
\hline Antes & 358 & 303 & 84,6 & 1 & - & $<0,000$ \\
\hline Depois & 354 & 328 & 92,6 & 1,09 & $1,03-1,15$ & \\
\hline Você faz c & & & & & & \\
\hline Antes & 344 & 220 & 63,9 & 1 & - & $<0,000$ \\
\hline Depois & 338 & 277 & 82,0 & 1,28 & $1,16-1,40$ & \\
\hline Já se embr & & & & & & \\
\hline Antes & 360 & 171 & 40,5 & 1 & - & $<0,000$ \\
\hline Depois & 354 & 251 & 59,5 & 1,66 & $1,31-2,11$ & \\
\hline Já se embr & & & & & & \\
\hline Antes & 245 & 77 & 31,4 & 1 & - & $<0,000$ \\
\hline Depois & 291 & 213 & 73,2 & 2,32 & $1,91-2,83$ & \\
\hline Já se embr & & & & & & \\
\hline Antes & 245 & 216 & 88,1 & 1 & - & $<0,021$ \\
\hline Depois & 291 & 273 & 93,8 & 1,06 & $1,00-1,12$ & \\
\hline Já se embr & & & & & & \\
\hline Antes & 245 & 5 & 2,0 & 1 & - & $<0,040$ \\
\hline Depois & 291 & 16 & 5,5 & 2,7 & $1,0-7,2$ & \\
\hline Já se embr & & & & & & \\
\hline Antes & 245 & 219 & 89,4 & 1 & - & $<0,000$ \\
\hline Depois & 291 & 150 & 51,5 & 2,65 & $1,81-3,86$ & \\
\hline Considera & & & & & & \\
\hline Antes & 361 & 74 & 20,5 & 1 & - & $<0,000$ \\
\hline Depois & 354 & 150 & 42,3 & 2,06 & $1,63-2,61$ & \\
\hline Considera & & & & & & \\
\hline Antes & 361 & 92 & 25,5 & 1 & - & $<0,321$ \\
\hline Depois & 354 & 79 & 22,3 & 0,87 & $0,67-1,13$ & \\
\hline $\begin{array}{l}\text { Estudante } \\
\text { desestimu }\end{array}$ & & & & & & \\
\hline Antes & 356 & 326 & 91,6 & 1 & - & $<0,057$ \\
\hline Depois & 350 & 333 & 95,1 & 0,96 & $0,92-1,00$ & \\
\hline Você recel & & & & & & \\
\hline Antes & 348 & 41 & 11,8 & 1 & - & $<0,000$ \\
\hline Depois & 351 & 156 & 44,4 & 0,26 & $0,19-0,36$ & \\
\hline $\begin{array}{l}\text { Durante a } \\
\text { do excess }\end{array}$ & & & & & & \\
\hline Antes & 344 & 258 & 75,0 & 1 & - & $<0,000$ \\
\hline Depois & 341 & 306 & 89,7 & 0,83 & $0,77-0,89$ & \\
\hline Sente-se a & & & & & & \\
\hline Antes & 361 & 69 & 19,1 & 1 & - & $<0,000$ \\
\hline Depois & 354 & 146 & 41,2 & 0,46 & $0,57-0,85$ & \\
\hline
\end{tabular}


álcool e entre aqueles que já se embriagaram, seja em festas da faculdade ou em festas de família $(p<0,000)$. Ao final do curso, os próprios alunos reconhecem o aumento no consumo de álcool $(\mathrm{p}<0,000)$. Mesmo que a maior parte dos estudantes tenha considerado importante receber treinamento específico sobre como desestimular o consumo excessivo de álcool, pouco mais de $44 \%$ receberam esse treinamento durante sua formação e apenas 41,2\% afirmaram se sentir aptos a orientar um paciente a parar de beber.

\section{DISCUSSÃO}

A grande mudança no decorrer do tempo foi o aumento importante no consumo de álcool, que já era considerado bem elevado no primeiro ano do curso. O número absoluto de estudantes que bebem aumentou, e esse aumento foi proporcionalmente mais elevado entre as mulheres. De acordo com Machado $^{7}$, a adesão a drogas foi mais encontrada em estudantes solteiros e do sexo masculino. Trabalhos mais recentes, porém, apontam que a diferenciação entre o consumo nos dois sexos é praticamente inexistente, como evidenciado também nesta pesquisa, pois as mulheres estão ingerindo bebida alcoólica no mesmo ritmo e quantidade dos homens ${ }^{7}$. Nesse caso, a principal diferença entre homens e mulheres permanece sendo o tipo de bebida de primeira escolha.

Além disso, o aumento do consumo de álcool ocorreu principalmente durante o período do internato, corroborando a hipótese de maior vulnerabilidade dos estudantes de Medicina, já que nesse período da graduação existe maior cobrança de suas responsabilidades como futuro profissional. De acordo com Gomes ${ }^{8}$, o consumo de álcool não ocorre apenas na população adulta, atinge também os adolescentes e jovens, que começam o seu consumo muito cedo. Portanto, com o fim da adolescência, fase em que os jovens deixam a escola para ingressar numa faculdade, alguns se vêem obrigados a deixar a casa dos pais e ir morar longe da família. Nesse momento, surgem novas amizades e oportunidades para viverem novas experiências, o que tem preocupado pais e pedagogos, pois o fato de morar sozinho aumenta a chance de experimentação tanto do álcool como de outras drogas ${ }^{9}$.

O aumento do consumo de álcool entre os estudantes ao longo desses quatro anos mostra que as festas - no ambiente acadêmico, no ambiente familiar ou comemorações ao longo do curso, como após uma prova - levam ao aumento do consumo e estimulam a manutenção do hábito pelos estudantes. Pesquisas realizadas em outras faculdades de Medicina não constataram relação estatisticamente significativa entre o hábito de beber e a condição de estar solteiro, apesar os solteiros constituam a maioria dos entrevistados ${ }^{2,6}$. Provavelmente, esta característica está relacionada ao fato de o curso de Medicina exigir muitos anos de formação e, por conseguinte, um longo caminho até se atingir a independência profissional ${ }^{10}$.

Além da elevada prevalência de consumo, há uma preocupação importante com o uso exagerado de álcool e embriaguez. Uma comparação entre as respostas dos acadêmicos de Medicina quando questionados sobre episódios de embriaguez indicou aumento significativo no decorrer do curso. Um estudo realizado recentemente numa universidade federal mostrou uma prevalência de consumo de álcool de $85 \%$ entre os estudantes da área da saúde ${ }^{2}$. O consumo de álcool tanto por estudantes de Medicina quanto por médicos é tido como uma ingestão em quantidades perigosas, embora os universitários ainda apresentem um consumo maior ${ }^{1}$.

$\mathrm{O}$ estudo do consumo de drogas por estudantes de Medicina foi realizado por vários pesquisadores, e a literatura mostra uma elevada taxa de consumo dessas substâncias por esses universitários, sendo o álcool a droga mais consumida. Pesquisas realizadas na Universidade Federal do Maranhão e na Faculdade de Medicina da Universidade Católica do Maule (Chile) mostraram a mesma porcentagem de estudantes que faziam consumo de álcool (64,5\%). Um estudo conduzido numa faculdade de Medicina do Sul de Minas Gerais constatou que $99 \%$ dos alunos já consumiram bebida alcoólica pelo menos uma vez na vida ${ }^{2,5,11}$. Este estudo reitera os dados da literatura ao mostrar que a prevalência do consumo de álcool é maior que a do consumo de tabaco entre os estudantes de Medicina e mais elevada que em outros cursos superiores ${ }^{12,13}$. Além disso, acrescenta aos fatos já existentes a evidência de que, mesmo com a discussão desse tema nas escolas médicas, o consumo e embriaguez aumentaram durante a formação.

Ao considerar os dois momentos da realização da pesquisa, verifica-se uma modificação de alguns aspectos relacionados ao consumo de álcool e tabaco entre os estudantes universitários em questão. Festas em que essas substâncias são mais consumidas estão relacionadas diretamente ao próprio ambiente acadêmico, seguidas por sua utilização no final de um dia estressante e após o período de provas ${ }^{14}$. Estes dados também foram obtidos nesta pesquisa, assim como o aumento estatisticamente significante no consumo durante festas de família. O consumo nessas situações pode estar associado a maior vulnerabilidade devido a fatores como estresse, carga horária extensiva e grandes responsabilidades impostas a esses estudantes.

Os universitários do curso de Medicina, apesar de serem futuros profissionais da saúde e receberem informações técnicas durante sua formação sobre os malefícios do consumo excessivo do álcool e do tabaco, apresentam índices eleva- 
dos de consumo dessas drogas. Muitos utilizam esses meios como "válvula de escape" para problemas psicológicos e de resiliência ocasionados por uma rotina estressante de estudos. Fatores como ansiedade, depressão, sair de sua cidade para cursar o ensino superior, morar sozinho e a própria concorrência existente no ambiente acadêmico acabam por interferir ainda mais no estado psíquico desses estudantes ${ }^{15}$. É comum ver a dificuldade que esses estudantes têm para reconhecer seus problemas e dificuldades, sobretudo no tocante ao uso abusivo de álcool e fumo.

Vale ressaltar ainda que o uso do álcool pode ser o gatilho inicial para o consumo de outras drogas, como o tabaco e substâncias ilícitas. A segunda droga cujo consumo é mais prevalente entre os estudantes de Medicina é o tabaco, apesar de atingir níveis mais baixos quando comparados aos do álcool. Um estudo realizado em 2012 nas 27 capitais brasileiras detectou uma prevalência de $12,1 \%$ de fumantes entre os maiores de 18 anos, sendo maior entre os homens (15,5\%) do que entre as mulheres $(9,2 \%)$. No entanto, em ambos os sexos, a frequência deste hábito foi menor antes dos 25 anos de idade ou após os 65 anos $^{16}$.

Foi observado um discreto aumento em relação ao consumo de tabaco. Foi avaliado que o fato de ser tabagista esteve associado a se embriagar e a experimentar bebida alcoólica em idades mais jovens e também houve uma quantidade maior de ingestão de bebida nessas pessoas. De modo geral, a prevalência do consumo do tabaco entre universitários de outras áreas é um pouco mais baixa em comparação aos estudantes da área da saúde, variando entre $8,1 \%$ e $14,7 \%$, e o cigarro industrializado foi o mais utilizado por estes estudantes ${ }^{16,17}$. Entre os acadêmicos da área da saúde, a prevalência de tabagismo variou entre $15 \%$ e $22 \%$.

Quando se analisa por sexo, uma pesquisa feita com 286 alunos da Polônia mostrou que os homens demonstram tendência maior no consumo de tabaco. No entanto, um estudo feito no Brasil com cerca de 450 estudantes não encontrou diferenças de prevalência entre os sexos. O hábito de fumar depende dos aspectos socioeconômicos, demográficos, culturais e até climáticos de cada país ${ }^{16}$. Foi percebido que a prevalência de consumo de álcool e tabaco foi maior no sexo masculino nos dois momentos da pesquisa, corroborando outros estudos em condições e públicos semelhantes.

Um dos fatores de risco que se mostrou importante para a manutenção do consumo de fumo pelos estudantes de Medicina foi a presença de outros familiares fumantes e morando na mesma casa, bem como a mudança no padrão de moradia entre os estudantes. Entre os fatores de risco para o consumo de fumo já evidenciados em outros trabalhos estão: morar so- zinho, mau desempenho acadêmico, reprovação em disciplinas do curso, uso prévio de álcool ${ }^{18}$.

A maior dificuldade do tabagismo não é a falta informações dos universitários, pois eles têm conhecimento das doenças causadas pelo tabaco. A questão, na maior parte das vezes, se refere ao fato de que dificilmente conseguem se perceber como possíveis portadores de doenças geradas pelo consumo do tabaco ${ }^{16}$.

Inúmeros fatores de risco podem contribuir para o uso indevido de drogas durante a formação no ensino superior. No curso de Medicina em particular, o estudante precisa se adaptar a uma nova realidade de ensino-aprendizagem, à aquisição de novas responsabilidades e à mudança de estilo de vida. A maior liberdade e a autonomia dos atos aliados à independência proporcionada pela maioridade tornam os universitários mais expostos ao consumo de drogas. Algumas das razões que podem estar relacionadas a essa fase da vida são a busca de novas experiências, a sensação juvenil e o desafio à estrutura familiar e social ${ }^{19,20}$. Dessa forma, pode surgir um conflito interpessoal, levando os jovens a buscar meios de tentar se ausentar dessa realidade.

Em ambos os momentos da pesquisa, os estudantes reconheceram a importância de receber treinamento sobre cessação do consumo do tabaco e álcool. De acordo com Guer$\mathrm{ra}^{16}$, os motivos de consumo de tabaco entre os universitários, principalmente da área da saúde, são imitação, curiosidade e aceitação social. É importante salientar que esses estudantes têm conhecimento dos malefícios ocasionados pelo tabaco, mas não abandonam o hábito. Tal comportamento pode ocorrer também devido a uma inadequação da grade curricular dos cursos da área da saúde, pois os universitários são apenas informados sobre os malefícios, quando deveriam ser sensibilizados de forma a se apropriar desse tema tão relevante para a sua saúde e para a saúde pública ${ }^{16}$. No setor da saúde, a formação dos profissionais para enfrentar problemas relacionados ao uso de drogas enfoca a dependência e não prioriza a prevenção, mostrando a deficiência da qualificação e a importância de propostas de formação profissional ${ }^{21}$. Assim, destaca-se a existência de uma lacuna na formação desses profissionais que precisa ser revista, para que consigam receber esses treinamentos em sua grade curricular.

A principal limitação do estudo ocorreu durante a aplicação dos questionários da segunda fase, quando os estudantes estavam no internato por conta do grande número de instituições a serem visitadas, o que dificultou bastante a coleta dos dados. 


\section{CONCLUSÃO}

Os dados deste estudo demonstram um aumento no consumo de álcool e tabaco entre os universitários do curso de Medicina no decorrer do curso. Assim, percebe-se a necessidade de adoção de medidas preventivas com o objetivo de reduzir os índices de alcoolismo e tabagismo nesta população.

Nos dois momentos do estudo, os estudantes consideraram importante ter treinamentos sobre as técnicas de cessação do hábito de fumar e consumo de álcool. O consumo excessivo de álcool e tabaco gera efeitos negativos à saúde, daí a necessidade de trabalhos de conscientização e promoção da saúde desses universitários. Esses futuros profissionais são formados para trabalhar na promoção e prevenção da saúde da população, e muitas vezes suas atitudes são exemplos seguidos pela comunidade.

\section{REFERÊNCIAS}

1. Magalhães LSP, Vernaglia TVC, Souza FAM, Chagas SV, Cruz MS. O Fenômeno das drogas na perspectiva dos estudantes de enfermagem: perfil do consumo, atitudes e crenças. Esc. Anna Nery. 2018 Feb; 22( 1 ): 1-8.doi: http:/ / dx.doi.org/10.1590/2177-9465-ean-2017-0205.

2. Pelicioli M, Barelli C, Gonçalves CBC, Hahn SR, Scherer JI. Alcohol consumption and episodic heavy drinking among undergraduate students from the health area of a Brazilian university. J. bras. psiquiatr.2017 July- Sep;66( 3 ): 150-156. doi: http:/ /dx.doi.org/10.1590/0047-2085000000164.

3. Ferraz L, Rebelatto SL, Schneider GC, Anzolin V. O uso de álcool e tabaco entre acadêmicos de uma universidade do sul do brasil. Rev. Bras. em Prom. da Saúde. 2017 Jan-Mar; 30(1):79-85.doi: http://www.redalyc.org/articulo. oa?id=40851313011.

4. Saraiva AGS, Chaves CMCB, Duarte JC, Amaral MOP. A dependência de tabaco em estudantes de enfermagem. Rev. Enf. Ref. 2017 Mar;serIV( 12 ): 9-18. doi: http:/ /dx.doi. org/10.12707/RIV16032.

5. Rabelo MO, Prates TEC, Sampaio CA. Consumo de álcool por estudantes da área da saúde: uma Revisão Sistemática da Literatura. Rev. Bras. de Pesq. em Ciênc. da Saúde. 2017 4(1): 01-08.

6. Pinheiro MA, Torres LF, Bezerra MS, Cavalcante RC, Alencar RD, Donato AC, et al. Prevalência e Fatores Associados ao Consumo de Álcool e Tabaco entre Estudantes de Medicina no Nordeste do Brasil. Rev. Bras. Educ. Méd.2017 41(2): 231-239. doi: https://dx.doi.org/10.1590/1981$-52712015 v 41 n 2 r b 20160033$.

7. Machado JNS, Finelli LAC, Jones KM, Soares WD. Consumo de álcool entre acadêmicos de Medicina. Rev. Bras. de Pesq. em Ciênc. da Saúde. 2016 2(2): 46-51.
8. Gomes BDMR, Alves JGB, Nascimento LC. Consumo de álcool entre estudantes de escolas públicas da Região Metropolitana do Recife, Pernambuco, Brasil. Cadernos de Saúde Pública. 2010 26:706-712. doi: http://dx.doi. org/10.1590/S0102-311X2010000400013.

9. Zepka BL, Oliveira GVL, Fonseca AD. Consumo alcoólico entre universitários (as) da área da saúde da Universidade Federal do Rio Grande/RS: subsídios para enfermagem. Esc. Anna Nery Rev. de Enfer. . 2012 Sep;16(3):530-535. doi: http:/ / www.redalyc.org/articulo.oa?id=127723305015.

10. Barbosa FL, Barbosa RL, Barbosa MCL, Aguiar DL, Figueiredo IA, Ribeiro AC, et al. Uso de álcool entre estudantes de Medicina da Universidade Federal do Maranhão. Rev. Bras. de Educ. Méd. 2013 Jan-Mar; 37(1), 89-95. doi: https:/ /dx.doi.org/10.1590/S0100-55022013000100013.

11. Tostes JG, Campos FP, Pereira LGR. Consumo de Álcool e Outras Drogas em uma Faculdade de Medicina do Sul de Minas Gerais/Consumption of Alcohol and Other Drugs in a Medical School in Southern Minas Gerais. Rev. Ciênc. em saúde 2016Jun; 6(2), 16-24. doi: http:/ /dx.doi. org/10.21876/rcsfmit.v6i2.484

12. Oliveira AF, Valente JG, Leite IC. Aspectos da mortalidade atribuível ao tabaco: revisão sistemática. Rev. Saúde Púb. 2008 Apr ; 42( 2 ), 335-345. doi: http:/ / dx.doi.org/10.1590/ S0034-89102008005000001

13. Amorim AVC, Kikko EO, Abrantes MM, Andrade VLÂ. Álcool e alcoolismo: estudo de prevalência entre discentes do curso de Medicina da UNIFENAS em Belo Horizonte-Minas Gerais. Rev Méd. Minas Gerais 200818(1), 16-23.

14. Pedrosa AAS, Camacho LAB, Passos SRL, Oliveira RVC. Consumo de álcool entre estudantes universitários. Cad. Saúde Púb. 2011 Aug ; 27( 8 ), 1611-1621. doi:http:/ /dx.doi. org/10.1590/S0102-311X2011000800016.

15. Joia, L. C.. Perfil do estilo de vida individual entre estudantes universitários. Rev. Movim., 3(1).

16. Guerra F, Costa C, Bertolini S, Marcon S, Parré J. Consumo de tabaco entre universitários: uma revisão sistemática Tobacco consumption among college students: a systematic review. Rev. de Pesq.: Cuidado é Fundamental Online . 2017 Apr; 9(2): 558-565. doi: http://dx.doi. org/10.9789/2175-5361.2017.v9i2.558-565

17. Silva BP, Sales CMM, França MG, Siqueira MM. Uso do tabaco entre estudantes de enfermagem de uma faculdade privada. SMAD-Rev. Elet. Saúde Mental Álcool e Drogas 2012 Aug; 8(2), 64-70. doi:http://dx.doi.org/10.11606/ issn.1806-6976.v8i2p64-70

18. Zettler EW, Nudelmann, LM, Cunha, DPD, Hilgert C, Mattos MD, Scholl M, et al. Prevalência do tabagismo entre 
estudantes de Medicina e fatores de risco associados. Rev. AMRIGS 2005 Jan-Mar;49(1), 16-19.

19. Mendonça AKRH, Jesus CVF, Figueiredo MBGA, Valido DP, Nunes MAP, Lima SO. Consumo de álcool e fatores associados ao binge drinking entre universitárias da área de saúde. Esc. Anna Nery 2018 Apr; 22(1). Doi: http:/ / dx.doi. org/10.1590/2177-9465-ean-2017-0096

20. Elicker E, Palazzo LDS, Aerts DRGDC, Alves GG, Câmara S. Uso de álcool, tabaco e outras drogas por adolescentes escolares de Porto Velho-RO, Brasil. Epidem. e Serv. de Saúde 2015 Jul-Sep;24(3), 399-410. Doi: https://doi. org/10.5123/S1679-49742015000300006.

21. Costa PHAD, Mota DCB, Cruvinel E, Paiva FSD, Gomide HP, Souza ICWD, et al. Training on alcohol and other drugs for health and social care professionals: report on experience. Interface-Comunicação, Saúde, Educação 2015 Apr-Jun; 19(53), 395-404. Doi: https://doi.org/10.1590/180757622014.0607.

\section{CONTRIBUIÇÃO DOS AUTORES}

Renata Allana da Costa Pereira, Brena Ferreira dos Santos e Marcelo de Almeida Pinheiro participaram do desenho do estudo, coleta de dados e redação do artigo. Ileana Pitombeira Gomes, Carlos Henrique Alencar e Luciano Pamplona de Góes Cavalcanti participaram do desenho do estudo, análise estatística dos dados, redação do artigo, discussão dos resultados e revisão da versão final. Todos os autores aprovaram a versão final do manuscrito.

\section{CONFLITO DE INTERESSES}

Os autores declaram não haver conflito de interesses.

\section{ENDEREÇO PARA CORRESPONDÊNCIA}

Ileana Pitombeira Gomes

Faculdade de Medicina da Universidade Federal do Ceará

Departamento de Saúde Comunitária

Rua Prof. Costa Mendes, 1608 - 5º andar-Fortaleza

CEP 60430-140 - CE

E-mail: ileana_gomes@yahoo.com.br 